\title{
Distinct Gene Expression Signatures in Lynch Syndrome and Familial Colorectal Cancer Type $X$
}

\author{
Mev Dominguez-Valentin ${ }^{1 * 9}$, Christina Therkildsen ${ }^{29}$, Srinivas Veerla ${ }^{1}$, Mats Jönsson ${ }^{1}$, Inge Bernstein ${ }^{2}$, \\ Åke Borg ${ }^{1}$, Mef Nilbert ${ }^{1,2}$
}

1 Department of Oncology, Institute of Clinical Sciences, Lund University, Lund, Sweden, 2 The Danish HNPCC Register, Clinical Research Centre and Department of Gastroenterology, Copenhagen University Hospital, Hvidovre, Denmark

\begin{abstract}
Introduction: Heredity is estimated to cause at least $20 \%$ of colorectal cancer. The hereditary nonpolyposis colorectal cancer subset is divided into Lynch syndrome and familial colorectal cancer type X (FCCTX) based on presence of mismatch repair (MMR) gene defects.

Purpose: We addressed the gene expression signatures in colorectal cancer linked to Lynch syndrome and FCCTX with the aim to identify candidate genes and to map signaling pathways relevant in hereditary colorectal carcinogenesis.

Experimental design: The $18 \mathrm{k}$ whole-genome c-DNA-mediated annealing, selection, extension, and ligation (WG-DASL) assay was applied to 123 colorectal cancers, including 39 Lynch syndrome tumors and 37 FCCTX tumors. Target genes were technically validated using real-time quantitative RT-PCR (qRT-PCR) and the expression signature was validated in independent datasets.

Results: Colorectal cancers linked to Lynch syndrome and FCCTX showed distinct gene expression profiles, which by significance analysis of microarrays (SAM) differed by 2188 genes. Functional pathways involved were related to G-protein coupled receptor signaling, oxidative phosphorylation, and cell cycle function and mitosis. qRT-PCR verified altered expression of the selected genes NDUFA9, AXIN2, MYC, DNA2 and H2AFZ. Application of the 2188-gene signature to independent datasets showed strong correlation to MMR status.

Conclusion: Distinct genetic profiles and deregulation of different canonical pathways apply to Lynch syndrome and FCCTX and key targets herein may be relevant to pursue for refined diagnostic and therapeutic strategies in hereditary colorectal cancer.
\end{abstract}

Citation: Dominguez-Valentin M, Therkildsen C, Veerla S, Jönsson M, Bernstein I, et al. (2013) Distinct Gene Expression Signatures in Lynch Syndrome and Familial Colorectal Cancer Type X. PLoS ONE 8(8): e71755. doi:10.1371/journal.pone.0071755

Editor: Ramon Andrade de Mello, Faculty of Medicine, University of Porto, Portugal

Received April 29, 2013; Accepted July 2, 2013; Published August 12, 2013

Copyright: (c) 2013 Dominguez-Valentin et al. This is an open-access article distributed under the terms of the Creative Commons Attribution License, which permits unrestricted use, distribution, and reproduction in any medium, provided the original author and source are credited.

Funding: The Danish and the Swedish Cancer Funds, from the Swedish Research Council, the Lundbeck foundation, the Nilsson Cancer Fund, the Kamprad Cancer Fund and the Hvidovre University Hospital, Denmark. The funders had no role in study design, data collection and analysis, decision to publish, or preparation of the manuscript.

Competing Interests: The authors have declared that no competing interests exist.

*E-mail: mev_dv@yahoo.com

9 These authors contributed equally to this work.

\section{Introduction}

Heredity is a major risk factor for colorectal cancer. Identification of individuals and families at increased risk allows for targeted surveillance, which has been shown to reduce morbidity and mortality from colorectal cancer [1,2]. Hereditary nonpolyposis colorectal cancer (HNPCG) accounts for 3-6\% of colorectal cancer. The syndrome is clinically classified according to the Amsterdam criteria, which require at least three HNPCGassociated cancers, two affected first-degree relatives and at least one individual diagnosed before age $50[3,4]$. Between one-third and half of the families that fulfill the Amsterdam criteria carry germline mismatch repair (MMR) gene mutations in $M L H 1$, MSH2, MSH6 or PMS2 and are referred to as having Lynch syndrome [5-7]. The remaining families with a yet unidentified genetic background show a predominance of colorectal cancer, frequent synchronous and metachronous adenomatous polyps and few extracolonic tumors and are referred to as familial colorectal cancer type X (FCCTX) [8,9]. Compared to Lynch syndrome, colorectal cancers linked to FCCTX develop later, predominantly occur in the distal colon and less often show the distinctive morphological features tumor-infiltrating lymphocytes, poor differentiation and mucin production characteristic of Lynch syndrome tumors $[8,10,11]$.

Data on the genetic features and tumorigenic pathways of FCCTX are scarce though genomic studies have demonstrated mean 6-8 copy number alterations with recurrent gains of $7 \mathrm{p}, 7 \mathrm{q}$, $8 \mathrm{q}, 13 \mathrm{q}, 20 \mathrm{p}$ and $20 \mathrm{q}$ and losses of $17 \mathrm{p}, 18 \mathrm{p}$ and $18 \mathrm{q}[9,12,13]$. Two studies have addressed gene expression profiles and mutation patterns in FCCTX tumors and have described similarities between FCGTX and sporadic MMR stable tumors [14,15]. In sporadic colorectal cancer, MMR defective tumors show distinct 
Table 1. Clinical characteristics of the colorectal cancer subsets.

\begin{tabular}{|c|c|c|c|c|}
\hline Characteristics & Lynch syndrome & FCCTX & $\begin{array}{l}\text { Sporadic MMR } \\
\text { deficient }\end{array}$ & $\begin{array}{l}\text { Sporadic MMR } \\
\text { proficient }\end{array}$ \\
\hline Number of tumors profiled & 39 & 37 & 26 & 21 \\
\hline Mean (range) age at onset & $53(25-86)$ & $58(33-88)$ & $74(62-86)$ & $69(51-83)$ \\
\hline Sex (\% female) & 54 & 38 & 54 & 57 \\
\hline Tumor location, proximal/distal (\%) & $77 / 23$ & $5 / 95$ & $81 / 19$ & $52 / 48$ \\
\hline Differentiation, high-moderate/low (\%) & $67 / 33$ & $92 / 8$ & $46 / 54$ & $86 / 14$ \\
\hline Tumor stage distribution (\%) & |:13, 11:51, $111: 36$ & |:8, II:43, III:49 & I:4, II:73, III:15 & I:0, II:43, III:57 \\
\hline
\end{tabular}

gene expression profiles with up-regulation of immunomodulatory genes, such as chaperones, cytokines and cytotoxic mediators, heat shock genes, major histocompatibility complex genes and apoptosis-related genes [16-18]. We applied global gene expression profiling in order to delineate candidate genes and differential involvement of pathways within the HNPCG subset of hereditary colorectal cancer with comparison between colorectal cancer linked to Lynch syndrome and FGCTX.

\section{Materials and Methods}

\section{Ethics Statement}

The project was ethically reviewed in Copenhagen, where a waiver for collection of tissues and clinical data was granted. All patients provided an informed consent for inclusion into the Danish HNPGC register during genetic counselling sessions. Ethical approval for the study was granted from the Scientific and Ethical Committee at The Capital Region of Copenhagen, Denmark (H-D-2007-0032).

\section{Sample Selection and RNA Extraction}

The national Danish HNPCG register was used to identify colorectal cancers from individuals with Lynch syndrome and FCCTX. Lynch syndrome was defined as families/individuals with disease-predisposing germline MMR gene mutations $(\mathrm{n}=16$ in MLH1, $\mathrm{n}=13$ in MSH2 and $\mathrm{n}=10$ in MSH6). Immunohistochemical MMR protein staining and microsatellite instability (MSI) analysis were performed as previously described $[11,13]$. FCCTX tumors were defined as tumors that developed in families fulfilling the Amsterdam criteria, but showed no disease-predisposing MMR gene mutations and had retained MMR function. Sporadic colorectal cancers were selected to represent MMR deficient and MMR proficient tumors from individuals without family history of cancer. Clinical data are summarized in Table 1. Compared to Lynch syndrome tumors, FCGTX tumors were more often located in the left side of the colon $(p<0.00001$, Fishers exact test) and showed high or moderate differentiation $(\mathrm{p}<0.00001$, Fishers exact test). Lynch syndrome and FCGTX did not differ as regards age at onset (mean 53 years versus 58 years) or tumor stage.

Non-necrotic tumor areas were macro-dissected and RNA extraction was performed from three $5-\mu \mathrm{m}$ sections of paraffinembedded tumor tissue $[11,13]$ using the High Pure RNA Paraffin Kit (Roche, Castle Hill, Australia) according to the manufacturer's instructions. RNA concentration was determined using a NanoDrop Spectrophotometer (NanoDrop Technologies, Wilmington, DE) and samples yielding sufficient RNA (200 ng) with 260/280 ratios $>1.8$ were selected.

\section{Gene Expression Profiling}

Gene expression analyses were performed at the SCIBLU Genomics Centre, Lund University, Sweden. The Illumina Beadarray (HumanWG-6 v4 Expression Beadchip, Illumina) system was used according to the manufacturer's instructions. Briefly, total RNA was converted to cDNA using biotinylated oligo-d $\mathrm{T}_{18}$ and random nonamer primers. Two assay-specific oligonucleotides were designed to interrogate a single contiguous $50 \mathrm{nt}$ sequence on each cDNA. Each of these oligonucleotides consisted of two parts: an upstream-specific oligonucleotide (USO) containing a $3^{\prime}$ gene-specific sequence and a $5^{\prime}$ universal PCR primer sequence (P1), while the downstream-specific oligonucleotide (DSO) contained a $5^{\prime}$ gene-specific sequence and a different $3^{\prime}$ universal PCR primer sequence (P2). Using this approach, a total of 24,526 oligonucleotide pairs (probes) were designed and pooled, which together constituted the whole genome (WG) DASL assay pool (DAP), corresponding to 18,626 unique genes, based on wellannotated content derived from the National Center for Biotechnology Information (NCBI) Reference Sequence Database (Build 36.2, Release 22). The DAP was then annealed to the targeted cDNAs, followed by enzymatic extension and ligation steps, as previously described [19]. Ligated products were PCR-amplified and labeled with a universal Cy3-coupled primer after which single-stranded labeled products were precipitated and hybridized to WG gene expression BeadChips as previously described [20]. BeadChips were then scanned on a BeadArray ${ }^{\text {TM }}$ Reader using BeadScan software (v4.2), during which fluorescence intensities were read and images extracted.

\section{Data Analysis}

Expression data were uploaded and processed in the GenomeStudio software (Illumina, San Diego, CA). Data were normalized using background correction, cubic spline method [21] and plate scaling. RefSeq features with a detection $P$ value of $\leq 0.01$ in at least $80 \%$ of the samples were used, leaving 9,218 features for further analysis. The data were uploaded into $\mathrm{MeV} v 4$ [22] where they were $\log 2$ transformed and median-centered across assays. Unsupervised clustering was performed on the total set of CRG samples using average linkage clustering with a Pearson correlation as similarity metric. Significance analysis of microarrays (SAM) [23] was used to identify significantly differentially expressed genes with a false-discovery rate (FDR) $\leq 5 \%$ [24]. Gene expression data are available in the NCBI Gene Expression Omnibus (GEO) (Accession number GSE36335). Biological pathways were identified using the web-based DAVID software with a FDR $\leq 5 \%$ [25]. 


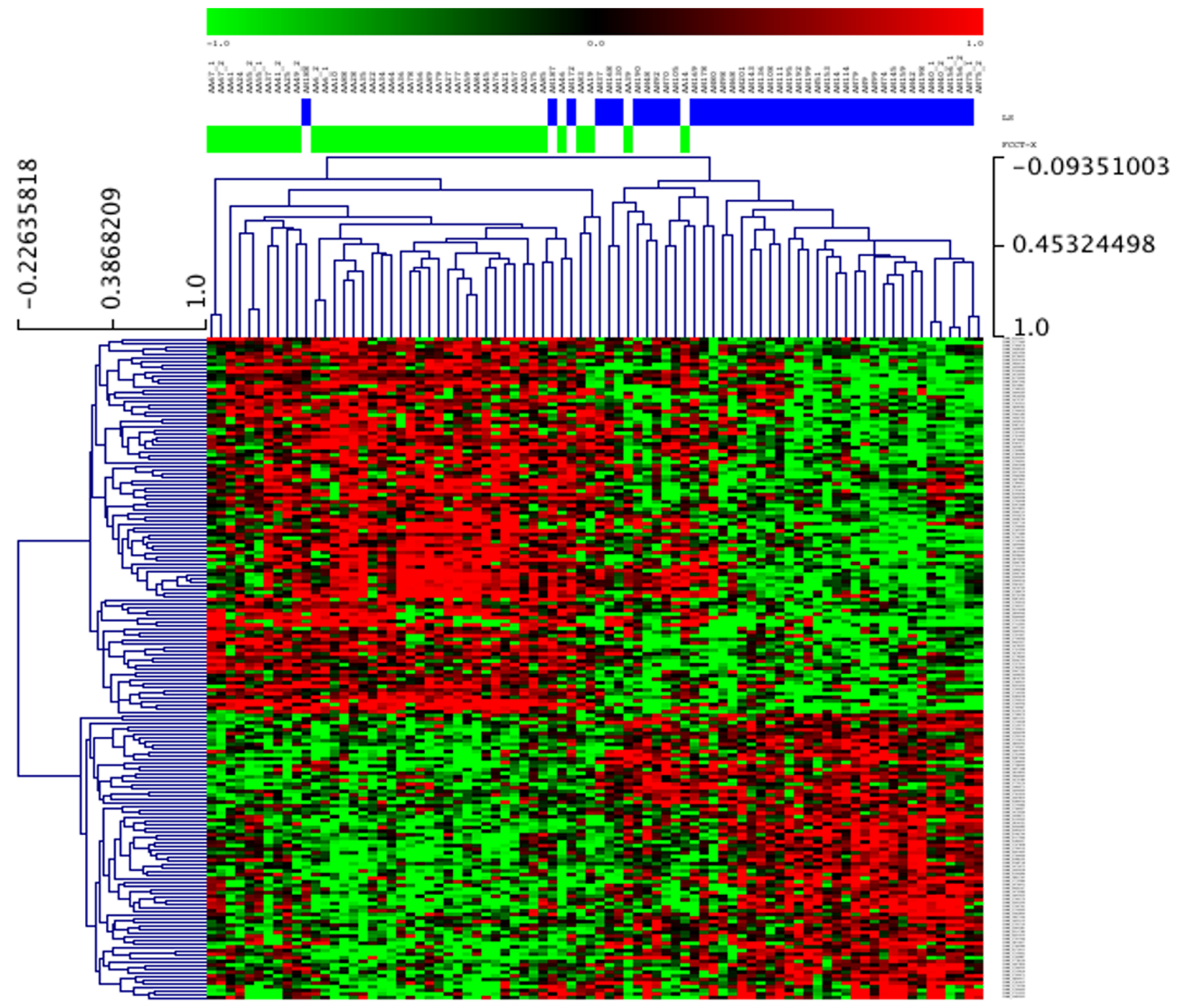

Figure 1. Clustering based on differentially expressed genes between FCCTX and Lynch syndrome tumors, identified by SAM analysis. The figure depicts the top-360 differentially expressed genes. Samples are arranged along the x-axis and show FCCTX tumors (green) and Lynch syndrome tumors (blue).

doi:10.1371/journal.pone.0071755.g001

Real Time Quantitative Reverse Transcription PCR (qRT$\mathrm{PCR})$

qRT-PCR was used to technically validate increased/decreased expression of 5 cancer-related genes with differential expression between the 4 colorectal cancer subsets. The genes were selected to represent major deregulated pathways and expression levels were investigated in 3 samples from each subtype. The $r R \mathcal{N} A 18 S$ was used as internal reference gene and normal colon sample as a calibrator. Gene-specific primers and taqman probe sets for each gene were obtained from Applied Biosystems (Applied Biosystems, Foster City, CA). Reverse transcription and PCR was performed using Quantitect reverse transcription kit and probe PCR kit, respectively (Qiagen, Heidelberg, Germany).

\section{Validation in External Data}

The hereditary gene expression signature was validated using the GSE4554 data set and the four largest batches of The Cancer Genome Atlas Network (TCGA) RNAseqv2 [26,27]. In order to resemble microarray data, the RPKM values of the TCGA were quantile-normalized, an offset of 32 was added, capped at 65,000, and genes were centered. Subsequently the data was adjusted for the batch variable. All data were imported into $\mathrm{MeV}$ v4, $\log 2$ transformed, genes were median-centered across assays and the $50 \%$ least-varying genes were removed. Unsupervised hierarchical clustering was performed as described above. A gene expression centroid was constructed by averaging the expression values of the samples in Lynch syndrome and FCGTX for each gene in order to establish a signature classifier for nearest centroid classification. A sample from the external datasets was assigned to either of the two hereditary subsets based on the maximum Pearson correlation of its centroid expression to the hereditary centroid values.

\section{Statistical Analysis}

Clinical association (tumor location, differentiation, age at onset and stage) in Lynch syndrome and FCCTX were determined using the Fisher's exact test (with significance set for $P<0.05$ ). The 
Table 2. Up-regulated signaling pathways in FCCTX and Lynch syndrome tumors.

\begin{tabular}{|c|c|c|c|c|}
\hline Tumor & Pathway & P-value & FDR (\%) & Genes \\
\hline FCCTX & Signaling by G-protein coupled receptor & $5 \times 10^{-4}$ & 0.589 & $\begin{array}{l}\text { GNAS, F2R, F2RL2, EDN1, EDNRA, GRM8, GNAZ, GNG11, } \\
\text { GNG12, HCRT, PTGER1, P2RY2, RAMP2, MC1R, TUBB3, VIP }\end{array}$ \\
\hline Tumor & Pathway & P-value & FDR (\%) & Genes \\
\hline \multirow[t]{2}{*}{ Lynch syndrome } & Cell cycle and mitosis & 0.001 & 1.416 & $\begin{array}{l}\text { CDC45, DNA2, E2F2, MIS12, MLF1IP, WEE1, ZWILCH, ANAPC4, } \\
\text { ANAPC5, BUB3, CASC5, CDC20, CENPM, CEP135, CCNB2, } \\
\text { CCNE1, CCNH, CDK2, CDKN1A, DHFR, FEN1, HSP9OAA1, } \\
\text { HSP9OAA2, KIF2C, KNTC1, MCM4, NUP37, PCNT, PCM1, PTTG1, } \\
\text { PTTG2, PLK4, POLE, POLD3, PSMD2, PSMD5, PSME1, PSME2, } \\
\text { PSMA4, PSMA5, PSMA6, PSMB2, PSMB4, PSMB6, PPP1CC, } \\
\text { PPP2CB, PPP2R5B, RFC5, RPA1, RRM2, STAG1, TYMS, } \\
\text { LOC442308, TUBB, TUBBP1, TUBBP2 }\end{array}$ \\
\hline & Oxidative phosphorylation & 0.003 & 3.508 & $\begin{array}{l}\text { ATP5L, ATP5A1, ATP5B, ATP5D, ATP6V1B2, ATP6V1A, NDUFA7, } \\
\text { NDUFA8, NDUFA9, NDUFB2, NDUFAB1, NDUFS2, NDUFV2, } \\
\text { TCIRG1, COX7B, COX7C, COX6A1, COX5A, PPA2, } \\
\text { LOC100130320, SDHD, SDHA, UQCR10, UQCRFS1, UQCRFSL1, } \\
\text { UQCRQ }\end{array}$ \\
\hline
\end{tabular}

doi:10.1371/journal.pone.0071755.t002

statistical analyses were performed using the statistical software package IBM SPSS Statistics 20 (SPSS, Chicago, IL, USA).

\section{Results}

Unsupervised hierarchical clustering analysis in the whole series (including 39 Lynch syndrome tumors, 37 FCGTX tumors, 21 sporadic MMR proficient tumors and 26 sporadic MMR deficient tumors) identified two major subgroups related to MMR status (Figure S1). The intrinsic MMR signature was strong with 3873 differentially expressed genes, of which 2159 (56\%) were upregulated in the MMR defective tumors and were related to 5 signaling pathways (Table $\mathrm{S} 1$ ).

In the hereditary tumor subset, SAM analysis identified 2188 differentially expressed genes between FCCTX and Lynch syndrome tumors (Figure 1). In FGCTX tumors, the G-protein coupled signaling pathway was up-regulated $(\mathrm{FDR}=0.6 \%)$, whereas Lynch syndrome tumors showed up-regulation of 2 pathways related to cell cycle and mitosis $(\mathrm{FDR}=1.4 \%)$ and oxidative phosphorylation (FDR $=3.5 \%$ ) (Table 2$)$.

The gene expression profiles of FCCTX tumors were closely related to those in sporadic MMR proficient colorectal cancers with genes involved in peptidyl-amino acid modification, enzyme linked receptor protein signaling and growth regulation. Likewise, Lynch syndrome and sporadic MMR defective tumors shared genes involved in cell cycle progression and immune response. Comparison between FCGTX and sporadic MMR proficient tumors revealed 4 differentially expressed genes, whereas comparison between FGCTX and sporadic MMR deficient tumors identified 3906 differentially expressed genes. Lynch syndrome tumors differed from sporadic MMR stable tumors by 415

A

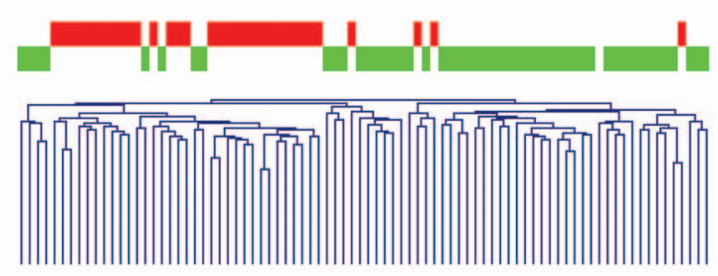

B

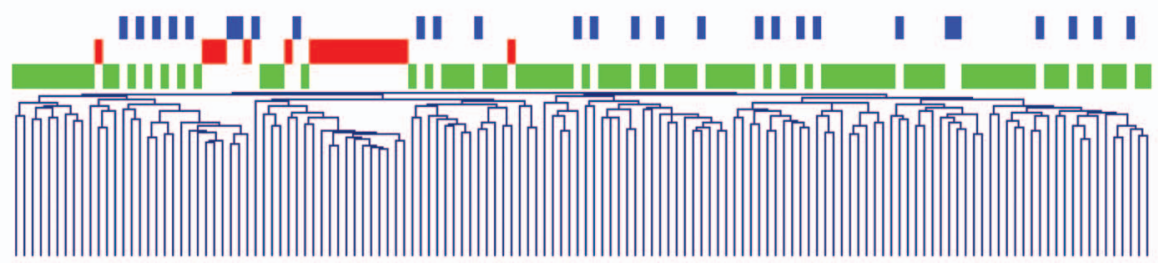

Figure 2. Unsupervised hierarchical clustering based on our 2188-gene signature applied to external data sets. a) Clustering based on GSE4554 and b) Clustering based on the four largest batches of the TCGA RNAseqv2 data sets. MMR proficient tumors (green), microsatellite-low tumors (blue) and MMR deficient tumors (red) along the x-axis.

doi:10.1371/journal.pone.0071755.g002 

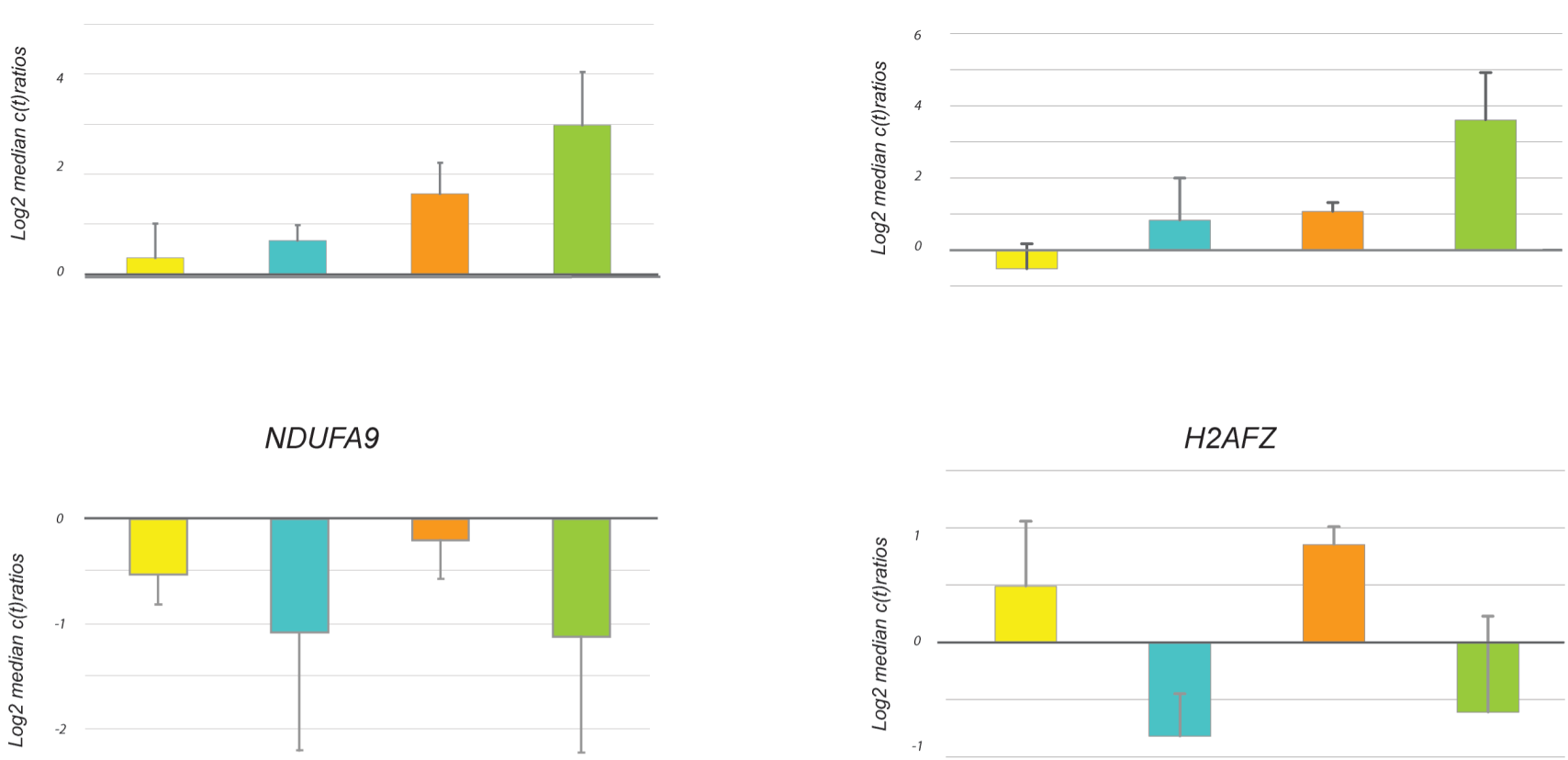

DNA2

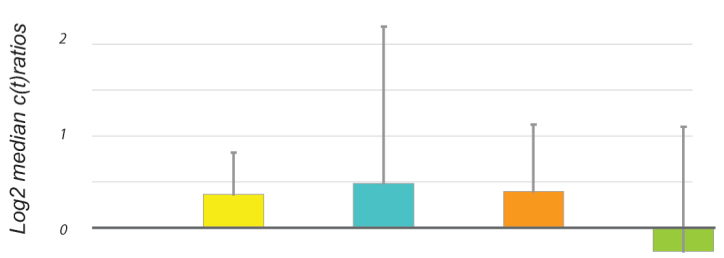

Sporadic MMR deficient tumors

Sporadic MMR proficient tumors

Lynch syndrome tumors

FCCTX tumors

Figure 3. qRT-PCR analysis of $\mathbf{5}$ target genes in the four different colorectal cancer subsets. Differential expression of MYC, NDUFA9, H2AFZ, AXIN2, and DNA2 was done for 12 representative samples ( 3 from each group) and qRT-PCR ratios were normalized to rRNA18S and median centered.

doi:10.1371/journal.pone.0071755.g003

differentially expressed genes and from sporadic MMR deficient tumors by 91 genes. Among sporadic tumors, 1384 genes differed MMR proficient and deficient tumors.

The genetic profiles were validated in independent datasets, i.e. the GSE4554 (Affymetrix microarray) and the TCGA (RNA sequencing) [26,27]. Both datasets mainly comprise sporadic colorectal cases, including $51 \mathrm{MMR}$ stable tumors and $33 \mathrm{MMR}$ defective tumors in the GSE4554 data set and 91 MMR stable, 19 MMR defective and 28 MMR-low stable tumors in the TCGA data set. Unsupervised hierarchical clustering based on our 2188 gene signature identified by us, resulted in two major groups related to MMR status. The hereditary signature correctly classified $82 \%$ and $80 \%$ of the MMR stable tumors as FCCTX and $100 \%$ and $94 \%$ of the MMR defective tumors as Lynch syndrome, respectively (Figure 2).

qRT-PCR-based technical validation was performed using 5 cancer-related genes representing deregulated pathways observed in MMR stable and deficient tumors, respectively (Figure 3). The results confirmed increased expression of $M Y C$ and $A X I N 2$ in FCGTX tumors, decreased expression of NDUFA9 in FCCTX tumors and in sporadic MMR proficient tumors and increased expression of $H 2 A F Z$ in MMR deficient tumors. No major differences were observed for $D \mathcal{N} A 2$.

\section{Discussion}

Within the HNPGC subset of hereditary colorectal cancer, we demonstrated distinctively different gene expression signatures in FCGTX and Lynch syndrome tumors, which differ by 2188 genes. Based on these gene expression differences, our data suggest that MMR status strongly influences genetic signatures, also within phenotypically similar families, which is in agreement to previous studies on sporadic colorectal cancer [16-18,27,36]. The FCCTX and Lynch syndrome profiles differed in 3 major cancer-related pathways, primarily related to cell cycle progression andmitosis, oxidative phosphorylation and $\mathrm{G}$ protein coupled receptor signaling, which have been correlated to colorectal carcinogenesis $[18,28,29,36]$.

The FCCTX tumors showed up-regulation of 1059 genes, several $(n=16)$ of which were related to the G-protein coupled 
receptor pathway (Table 2). A candidate target herein includes the GNAS gene, which encodes for the $\mathrm{G}_{\alpha}$-subunit, and is located in the 20q13.32 region that has been found to be gained in FCGTX tumors [9,12,13]. Activating mutations in GNAS have been described in CRC and are suggested to promote tumorigenesis through activation of the Wnt and ERK1/2 MAPK signaling pathways [28,29]. The potential tumorigenic mechanism is unknown and the hot-spot mutation GNAS c.601G>T has not been observed in FCCTX tumors [12]. Also other candidate genes involved in proliferation and migration, e.g. $C D H 26, S R C$ and ASIP are located in 20q [30,31]. The FCCTX tumors also showed up-regulation of PTGER1, which encodes for the EP1 receptor that may promote proliferation and colorectal tumor development through altered function of prostaglandin E2 ( $\left.\mathrm{PGE}_{2}\right)$ [32-34].

Lynch syndrome tumors showed up-regulation of 1129 genes, e.g. genes involved in $\mathrm{G}_{1} / \mathrm{S}$ transition (CCNE, CCNH, E2F2 and CDK2), DNA replication (CDC45, RPA1, RFC5, MCM4 and $P O L D 3)$ and chromosomal organization and mitosis (CCNB2, MLF1IP, CASC5, KIF2C and PLK4), which is in line with previous findings (Table 2) $[16,18,35,36]$. Up-regulation of e.g. WEE1, $C D K \mathcal{N} 1 A$ and FANCD2 were also observed in our study and have also been reported by other investigators, which may suggest involvement of the cell cycle checkpoint machinery $[37,38]$. Overexpression of checkpoint proteins have previously been linked to Lynch syndrome and abrogation of the checkpoint machinery has been shown to sensitize MMR deficient tumor cells towards chemotherapy $[39,40]$. Also genes involved in the oxidative phosphorylation pathway, e.g. the ATP synthase subunit genes and complex I and III subunit genes were among those upregulated in Lynch syndrome tumors. Down-regulation of ATP synthase subnit genes and complex I, III and V genes have been correlated to metastastic colorectal cancer and may explain the more aggressive tumor development and poor prognosis observed in MMR proficient tumors [11,18,41].

In colorectal cancer, MMR status is a major discriminator related to distinctively different gene expression profiles, which is supported by the 3873 differentially expressed genes in our series of MMR deficient and proficient tumors [15,16,27]. The importance of MMR status was observed also when we applied the 2188-gene signature that discriminated between Lynch syndrome and FCCTX to two independent data sets mainly containing sporadic tumors. The signature correctly defined $80-$ $82 \%$ of the MMR proficient and $94-100 \%$ of MMR deficient

\section{References}

1. Mecklin JP, Järvinen HJ (2005) Surveillance in Lynch syndrome. Fam Cancer 4: 267-271. Review.

2. Balmaña J, Castells A, Cervantes A, ESMO Guidelines Working Group (2010) Familial colorectal cancer risk: ESMO Clinical Practice Guidelines. Ann Oncol 5: v78-81.

3. Vasen HF, Mecklin JP, Khan PM, Lynch HT (1991) The international collaborative group on hereditary non-polyposis colorectal cancer (ICGHNPCC). Dis Colon Rectum 34: 424-425.

4. Vasen HF, Watson P, Mecklin JP, Lynch HT (1999) New clinical criteria for hereditary nonpolyposis colorectal cancer (HNPCC, Lynch syndrome) proposed by the International Collaborative group on HNPCC. Gastroenterology 116: 1453-1456.

5. Lynch HT, Lynch PM (2005) Molecular screening for the Lynch syndromebetter than family history? N Engl J Med 352: 1920-1922.

6. Jass JR (2006) Colorectal cancer: a multipathway disease. Crit Rev Oncog 12: 273-287. Review.

7. Vasen HF, Möslein G, Alonso A, Bernstein I, Bertario L, et al. (2007) Guidelines for the clinical management of Lynch syndrome (hereditary non-polyposis cancer). J Med Genet 44: 353-362.

8. Lindor NM, Rabe K, Petersen GM, Haile R, Casey G, et al. (2005) Lower cancer incidence in Amsterdam-I criteria families without mismatch repair deficiency: familial colorectal cancer type X. JAMA 293: 1979-1985.

9. Abdel-Rahman WM, Ollikainen M, Kariola R, Järvinen HJ, Mecklin J, et al. (2005) Comprehensive characterization of HNPCG-related colorectal cancers tumors (Figure 2). Several heat shock proteins and immune response genes (Table S1) were up-regulated in the MMR defective tumors, which supports involvement of immune-response mechanisms, irrespective of whether the tumor was caused by germline or somatic MMR gene inactivation [16-18,42,43]. In sporadic as well as hereditary MMR proficient tumors several genes in the Wnt signaling were up-regulated, which fits well with its central role in colorectal tumorigenesis (Table S1) [44,45].

In conclusion, hereditary colorectal cancers within the HNPCG subset show distict genetic profiles with 2188 differentially expressed genes between Lynch syndrome and FCCTX. These data pinpoint genes and pathways relevant to further pursue for refined diagnostics and therapeutics in hereditary colorectal cancer.

\section{Supporting Information}

Figure S1 Unsupervised hierarchical clustering of the entire dataset. The dendogram shows the spontaneous clustering of 123 colorectal cancers into two major clusters related to MMR status. MMR proficient tumors (green), including FCCTX tumors and sporadic MMR proficient tumors and MMR deficient tumors (blue), including Lynch syndrome tumors and sporadic MMR deficient tumors.

(TIF)

Table S1 Signaling pathways up-regulated in MMR proficient and MMR deficient tumors.

(XLS)

\section{Acknowledgments}

We thank Eva Rambech and Martin Lauss, Department of Oncology, Institute of Clinical Sciences, Lund University and Anja Nissen, Clinical Research Centre, Copenhagen University Hospital, Hvidovre for technical help and assistance and Sabrina Da Silva, Department of Medicine and Oncology, Sir Mortimer B, Davis-Jewish General Hospital, McGill University, Montreal, Canada for statistical support.

\section{Author Contributions}

Conceived and designed the experiments: MDV CT MN SV MJ AB IB. Performed the experiments: MDV MJ. Analyzed the data: MDV SV. Contributed reagents/materials/analysis tools: MDV CT SV MJ IB AB MN. Wrote the paper: MDV CT MN. reveals striking molecular features in families with no germline mismatch repair gene mutations. Oncogene 24: 1542-1551.

10. Llor X, Pons E, Xicola RM, Castells A, Alenda C, et al. (2005) Differential features of colorectal cancers fulfilling Amsterdam criteria without involvement of the mutator pathway. Clin Cancer Res 11: 7304-7310.

11. Klarskov L, Holck S, Bernstein I, Nilbert M (2012) Hereditary colorectal cancer diagnostics: morphological features of familial colorectal cancer type $\mathrm{X}$ versus Lynch syndrome. J Clin Pathol 65: 352-356.

12. Middeldorp A, Van Eijk R, Oosting J, Forte GI, van Puijenbroek M, et al. (2012) Increased frequency of $20 \mathrm{q}$ gain and copy-neutral loss of heterozygosity in mismatch repair proficient familial colorectal carcinomas. Int J Cancer 130: 837-846.

13. Therkildsen C, Jönsson G, Dominguez-Valentin M, Nissen A, Rambech E, et al. (2012) Gain of chromosomal region 20q and loss of 18 discriminates between Lynch syndrome and familial colorectal cancer. Eur J Cancer. Dec 11. doi:pii: S0959-8049(12)00896-9.10.1016/j.ejca.2012.11.011.

14. Lee WS, Seo G, Shin HJ, Yun SH, Yun H, et al. (2008) Identification of differentially expressed genes in microsatellite stable HNPCC and sporadic colon cancer. J Surg Res 144: 29-35.

15. Sánchez-de-Abajo A, de la Hoya M, van Puijenbroek M, Tosar A, López-Asenjo JA, et al. (2007) Molecular analysis of colorectal cancer tumors from patients with mismatch repair proficient hereditary nonpolyposis colorectal cancer suggests novel carcinogenic pathways. Clin Cancer Res 13: 5729-5735. 
16. Kim H, Nam SW, Rhee H, Shan Li L, Ju Kang H, et al. (2004) Different gene expression profiles between microsatellite instability-high and microsatellite stable colorectal carcinomas. Oncogene 23: 6218-6225.

17. Banerjea A, Ahmed S, Hands RE, Huang F, Han X, et al. (2004) Colorectal cancers with microsatellite instability display mRNA expression signatures characteristic of increased immunogenicity. Mol Cancer 3: 21.

18. Bertucci F, Salas S, Eysteries S, Nasser V, Finetti P, et al. (2004) Gene expression profiling of colon cancer by DNA microarrays and correlation with histoclinical parameters. Oncogene 23: 1377-1391.

19. Fan JB, Yeakley JM, Bibikova M, Chudin E, Wickham E, et al. (2004) A versatile assay for high-throughput gene expression profiling on universal array matrices. Genome Res 14: 878-885.

20. Kuhn K, Baker SC, Chudin E, Lieu MH, Oeser S, et al. (2004) A novel, highperformance random array platform for quantitative gene expression profiling. Genome Res 14: 2347-2356.

21. Workman C, Jensen LJ, Jarmer H, Berka R, Gautier L, et al. (2002) A new nonlinear normalization method for reducing variability in DNA microarray experiments. Genome Biol 30: 3(9): research0048.

22. Saeed AI, Sharov V, White J, Li J, Liang W, et al. (2003) TM4: a free, opensource system for microarray data management and analysis. Biotechniques 34 : 374-378.

23. Tusher VG, Tibshirani R, Chu G (2001) Significance analysis of microarrays applied to the ionizing radiation response. Proc Natl Acad Sci USA 24: 5116 5121. Erratum in: Proc Natl Acad Sci USA 28: 10515.

24. Benjamini Y, Drai D, Elmer G, Kafkafi N, Golani I (2001) Controlling the false discovery rate in behavior genetics research. Behav Brain Res 125: 279-284.

25. Huang da W, Sherman BT, Lempicki RA (2009) Systematic and integrative analysis of large gene lists using DAVID bioinformatics resources. Nat Protoc 4: 44-57.

26. Cancer Genome Atlas Network (2012) Comprehensive molecular characterization of human colon and rectal cancer. Nature 487: 330-337.

27. Watanabe T, Kobunai T, Toda E, Yamamoto Y, Kanazawa T, et al. (2006) Distal colorectal cancers with microsatellite instability (MSI) display distinct gene expression profiles that are different from proximal MSI cancers. Cancer Res 66: 9804-988.

28. Sjöblom T, Jones S, Wood LD, Parsons DW, Lin J, et al. (2006) The consensus coding sequences of human breast and colorectal cancers. Science 314: 268-274.

29. Wilson CH, McIntyre RE, Arends MJ, Adams DJ (2010) The activating mutation R201C in GNAS promotes intestinal tumourigenesis in $\mathrm{Apc}(\mathrm{Min} /+)$ mice through activation of Wnt and ERK1/2 MAPK pathways. Oncogene 29: $4567-4575$.

30. Hu Y, Fang C, and Xu Y (2005) The effect of isoforms of the cell polarity protein, human ASIP, on the cell cycle and Fas/FasL-mediated apoptosis in human hepatoma cells. Cell Mol Life Sci 62: 1974-1983.

31. Jin YT, Ying XX, Hu YH, Zou Q Wang HY, et al. (2008) aPKC inhibitors might be the sensitizer of chemotherapy and adoptive immunotherapy in the treatment of hASIPa-overexpressed breast cancer. Oncol Res 17: 59-68.
32. Watanabe K, Kawamori T, Nakatsugi S, Ohta T, Ohuchida S, et al. (1999) Role of the prostaglandin E receptor subtype EP1 in colon carcinogenesis. Cancer Res 59: 5093-5096.

33. Kim SH, Park YY, Kim SW, Lee JS, Wang D, et al. (2011) ANGPTL4 induction by prostaglandin E2 under hypoxic conditions promotes colorectal cancer progression. Cancer Res 71: 7010-7020.

34. Greenhough A, Smartt HJ, Moore AE, Roberts HR, Williams AC, et al. (2009) The COX-2/PGE2 pathway: key roles in the hallmarks of cancer and adaptation to the tumour microenvironment. Carcinogenesis 30: 377-386. Review.

35. Yuan Z, Sotsky KT, Weber TK (2003) Differential expression of DOC-1 in microsatellite-unstable human colorectal cancer. Oncogene 22: 6304-6310.

36. Dunican DS, McWilliam P, Tighe O, Parle-McDermott A, Croke DT (2002) Gene expression differences between the microsatellite instability (MIN) and chromosomal instability (CIN) phenotypes in colorectal cancer revealed by highdensity hybridization. Oncogene 21,3253-3257.

37. Sinicrope FA, Roddey G, Lemoine M, Ruan S, Stephens LC et al. (1998). Loss of p21WAF1/Cipl protein expression accompanies progression of sporadic colorectal neoplasms but not hereditary nonpolyposis colorectal cancers. Clin Cancer Res 4: 1251-1261.

38. Duldulao MP, Lee W, Le M, Chen Z, Li W et al. (2012). Gene expression variations in microsatellite stable and unstable colon cancer cells. J Surg Res 174: $1-6$.

39. Jardim MJ, Wang Q Furumai R, Wakeman T, Goodman B.K, Wang XF (2009b). Reduced ATR or Chk1 expression leads to chromosome instability and chemosensitization of mismatch repair-deficient colorectal cancer cells. Mol Biol Cell 20: 3801-3809.

40. Pires IM, Ward TH, Dive G (2010) Oxaliplatin responses in colorectal cancer cells are modulated by CHK2 kinase inhibitors. Br J Pharmacol 159: 13261338.

41. Benatti P, Roncucci L, Ganazzi D, Percesepe A, Di GC et al. (2001). Clinical and biologic heterogeneity of hereditary nonpolyposis colorectal cancer. Int J Cancer 95: 323-328.

42. Belt EJ, Brosens RP, Delis-van Diemen PM, Bril H, Tijssen M, et al. (2012) Cell Cycle Proteins Predict Recurrence in Stage II and III Colon Cancer. Ann Surg Oncol 3: S682-692.

43. Ogino S, Nosho K, Irahara N, Meyerhardt JA, Baba Y, et al. (2009) Lymphocytic reaction to colorectal cancer is associated with longer survival, independent of lymph node count, microsatellite instability, and CpGisland and methylator phenotype. Clin Cancer Res 15: 6412-6420.

44. Yang M, Zhong WW, Srivastava N, Slavin A, Yang J, et al. (2005) G proteincoupled lysophosphatidic acid receptors stimulate proliferation of colon cancer cells through the $\{$ beta $\}$-catenin pathway. Proc Natl Acad Sci USA 102: 60276032.

45. Malbon CC (2005). Beta-catenin, cancer, and G proteins: not just for frizzleds anymore. Sci STKE 292: pe35. Review. 